\title{
Análisis de Sensibilidad de una Metodología de Decisión Multicriterio desarrollada para la Selección de Tipologías de Estructuras de Contención en una Autovía Urbana
}

\author{
Belén Muñoz \\ Prof. Asociado, Universidad Politécnica de Madrid, España \\ Manuel G. Romana \\ Prof. Titular de Universidad, UPM, España \\ Javier Ordóñez \\ Prof. Titular de Universidad, Universidad de Granada, España
}

\section{RESUMEN}

Los métodos de decisión multicriterio son una herramienta que reduce la subjetividad en la toma de decisiones mediante la creación de una serie de filtros de selección y ayuda a la elección entre alternativas complejas. Permite a los proyectistas estructurar la información de forma racional y poder exponerla de forma clara, así como la identificación precisa y ordenada todos los elementos que determinan que un diseño sea el idóneo, y al mismo tiempo se consideren y valoren todas las alternativas posibles que da solución al problema. Pueden tener propósitos diferentes en cada una de las etapas de selección de alternativas, en primer lugar puede ayudar a analizar el problema, tarea u objetivo al descomponerlo en un número finito de requisitos, y una vez establecido los requisitos ayuda a su ordenación por la importancia relativa o peso de cada criterio para cada alternativa. Frente a estas ventajas, presentan un inconveniente muy importante que es la subjetividad a la hora de asignar pesos a cada criterio y la valoración de las diferentes alternativas por el decisor.

En la presente comunicación se realiza un análisis de sensibilidad que valida la metodología desarrollada en el marco de una investigación que establece un proceso sistemático de decisión a partir de la aplicación de forma secuencial de varios métodos de decisión multicriterio, de manera que se reduzca en todo lo posible la subjetividad asociada a cualquier proceso de decisión. El análisis de sensibilidad determina cómo varía la selección de alternativas cuando varía la importancia relativa de los criterios o requerimientos de proyecto considerados como determinantes en el proceso de selección.

\section{INTRODUCCIÓN}

Los procesos de decisión están presentes en todas las actividades de la vida cotidiana. A través de la decisión intentamos dar solución a un determinado problema de la forma más adecuada, pero es conveniente recordar que este proceso es complejo y se desarrolla en un ambiente de incertidumbre.

Los métodos de decisión son herramientas que reducen la subjetividad en la toma de 
decisiones mediante la creación de una serie de filtros de selección y ayuda a la elección entre alternativas complejas. Puede servir para un propósito diferente en cada etapa, en primer lugar puede ayudar a analizar el problema, tarea u objetivo al descomponerlo en un número finito de requisitos, y una vez establecido los requisitos ayuda a su ordenación por la importancia relativa o peso de cada criterio para cada alternativa, (Muñoz et al, 2014).

Los métodos de decisión necesitan una fase de estructuración previa muy laboriosa y compleja que, para algunos autores, puede representar cerca del $80 \%$ del total del problema. Esta fase incluye la identificación de los objetivos del decisor, la identificación de alternativas viables, la selección de criterios (no siempre evidentes), la cuantificación de variables subjetivas, entre otras etapas que dependen de cada problema abordado, (Correia Baptista et al., 2004). Y lo que es más importante, determinar la importancia relativa de un criterio sobre el resto para obtener una ponderación objetiva.

En la mayoría de los problemas de decisión, con la existencia de múltiples criterios en conflicto, se recomienda la detección del conjunto de soluciones eficientes en lugar de la determinación de la solución óptima. Debido a la incertidumbre en los datos, procedimientos y enfoques empleados para su resolución sugieren realizar un estudio del comportamiento del proceso de toma de decisiones lo más completo posible, (Moreno-Jiménez, 1998).

No hay consenso en cómo determinar la "calidad" de un método de decisión y de la fiabilidad de los resultados obtenidos. En este sentido, el análisis de sensibilidad se puede definir como la estabilidad o comportamiento de la solución ante pequeñas modificaciones en las preferencias ocurridas durante el proceso de resolución o ante pequeñas modificaciones en los valores tomados para los parámetros, es lo que algunos autores consideran como eficiencia del método de decisión multicriterio. Mientras que la validez evalúa la calidad integral del proceso de decisión, o lo que sería la efectividad. Y por último, la robustez es el análisis del comportamiento del método de decisión de manera que se evalúe la posibilidad de cambio de rango entre las alternativas cuando se añaden o eliminan aspectos relevantes (alternativas, criterios, dependencias, etc.) respecto a los iniciales, mediría la eficacia.

En la bibliografía hay otras definiciones del análisis de sensibilidad, siendo éste un proceso sistemático utilizado para explorar cómo una solución considerada como "óptima" en el sentido paretiano, responde a los cambios introducidos en las condiciones de partida, las cuales típicamente serán o son valores conocidos que podrán variar en el futuro o parámetros cuyos valores estarán expuestos a cuestionamiento. De este modo, el análisis se sustenta alrededor del supuesto previo de que la optimización es el escenario principal, con la incertidumbre considerada como un factor potencialmente perjudicial. El análisis tiene como objetivo estudiar y descubrir el grado de sensibilidad de la solución óptima ante cambios en los factores esenciales. Una solución insensible es considerada como ventajosa, (Fernández y Escribano). 


\section{APLICACIÓN DE METODOLOGÍA PARA LA SELECCIÓN DE ESTRUCTURA DE CONTENCIÓN.}

Se plantea una metodología para la resolución del problema, como combinación de dos métodos de decisión multicriterio, Proceso Jerárquico de Análisis (AHP) y método VIKOR, (Muñoz et al., 2014).

En primer lugar y mediante el Proceso Jerárquico de Análisis, AHP, se calcula el autovector de pesos para los criterios que son determinantes en la solución más idónea, mediante comparación pareada de los mismos. Hay que indicar que el autovector de pesos dependerá de cada proyecto, es decir, un criterio puede tener una mayor importancia relativa respecto al resto dependiendo de las características del proyecto. Es necesario recordar, que AHP mide la inconsistencia global de los juicios mediante la Proporción de Consistencia, que se expresa como el cociente entre el Índice de Consistencia y el Índice Aleatorio, y debe ser inferior al 10\%, (Saaty, 1994). De igual forma, mediante AHP se determina el "comportamiento" de cada alternativa para cada uno de los criterios cualitativos que intervienen en el proceso de decisión, con el objetivo de obtener una valoración cuantitativa para un criterio cualitativo.

Posteriormente, se aplica el método VIKOR para la selección de la tipología más adecuada, a partir de un ranking de alternativas que nos proporcionan una o varias soluciones de compromiso. El método VIKOR es apropiado para resolver problemas de decisión con criterios en conflicto y no conmensurables, es decir, con distintas unidades, o en el caso de que haya criterios cuantitativos y cualitativos. La solución de compromiso viene determinada como aquella que se encuentra a una distancia más corta de la solución ideal, (Opricovic y Tzeng, 2007).

\section{APLICACIÓN A UNA ESTRUCTURA DE CONTENCIÓN EN UNA AUTOVIA URBANA.}

Para el análisis y selección, se consideran cuatro tipologías de muros como alternativas: muro de hormigón armado, muro jardinera, muro vegetal reforzado con geotextil y muro de escollera. Y se consideran como criterios determinantes en el proceso de decisión los siguientes: coste en $€ / \mathrm{m}$, rendimiento de construcción, $\mathrm{m} / \mathrm{día}$, integración paisajística, cultura, entendida esta como frecuencia de construcción, y por último, necesidades de conservación y mantenimiento. Hay que indicar que los diferentes muros tienen una altura media de cuatro metros, (Muñoz, et al., 2014).

Para la aplicación de la metodología, y para poder realizar iteraciones del proceso de forma automática, todo el proceso se ha programado en MATLAB®. De manera que partiendo, de la siguiente matriz de alternativas:

\begin{tabular}{|l|l|l|l|l|}
$\begin{array}{l}\text { Coste } \\
(€ / \mathrm{m})\end{array}$ & $\begin{array}{l}\text { Rendimiento } \\
\mathrm{m} / \text { día }\end{array}$ & $\begin{array}{l}\text { Integración } \\
\text { Paisajística }\end{array}$ & Cultura & $\begin{array}{l}\text { Necesidad de } \\
\text { Conservación }\end{array}$ \\
\hline
\end{tabular}




\begin{tabular}{|l|r|r|r|r|r|}
\hline \multicolumn{1}{|l|}{$\begin{array}{l}\text { Muro Hormigón } \\
\text { Armado }\end{array}$} & 870 & 1,5 & 0,041 & 0,506 & 0,047 \\
\hline Muro Jardinera & 640 & 2,48 & 0,099 & 0,257 & 0,316 \\
\hline Muro Vegetal & 430 & 2,75 & 0,608 & 0,065 & 0,564 \\
\hline Muro de Escollera & 608 & 2,8 & 0,251 & 0,172 & 0,073 \\
\hline
\end{tabular}

Tabla 1: Matriz de alternativas.

Teniendo en cuenta que al tratarse de una autovía urbana en servicio, es necesario priorizar el plazo y las necesidades de conservación para alterar lo menos posible el tráfico, tanto durante la construcción como durante la fase de explotación. Al aplicar AHP, para determinar por comparaciones pareadas el vector de pesos que cumple el Índice de Consistencia, y con las anteriores premisas, se obtiene el siguiente vector de pesos: $\mathrm{WC}=(0,17 ; 0,44 ; 0,04 ; 0,24 ; 0,11)$.

De esta forma, como resultado del método de decisión de VIKOR, se obtienen los siguientes valores y ranking.

\begin{tabular}{|c|c|c|c|c|}
\hline & $\begin{array}{l}\text { Muro Hormigón } \\
\text { Armado }\end{array}$ & Muro Jardinera & $\begin{array}{l}\text { Muro } \\
\text { Vegetal }\end{array}$ & $\begin{array}{l}\text { Muro de } \\
\text { Escollera }\end{array}$ \\
\hline $\mathbf{S}_{\mathbf{j}}$ & 0,6529 & 0,4203 & 0,3638 & 0,2823 \\
\hline $\mathbf{R}_{\mathbf{j}}$ & 0,4350 & 0,1330 & 0,2361 & 0,1788 \\
\hline $\mathbf{Q}_{\mathbf{j}}$ & 1,0000 & 0,1861 & 0,2804 & 0,0754 \\
\hline
\end{tabular}

Tabla 2: Valores de Sj, Rj y Qj, resultado método VIKOR.

\begin{tabular}{l|c|c|c|}
\multicolumn{1}{c|}{} & $\mathbf{Q}$ & $\mathbf{S}$ & $\mathbf{R}$ \\
\hline Muro Escollera & 0,0754 & 0,2823 & 0,1788 \\
\hline Muro Jardinera & 0,1861 & 0,4203 & 0,1330 \\
\hline Muro vegetal & 0,2804 & 0,3638 & 0,2361 \\
\hline Muro Hormigón Armado & 1,0000 & 0,6529 & 0,4350 \\
\hline
\end{tabular}

Tabla 3: Ranking de alternativas según método VIKOR.

Hay que hacer notar que $\mathrm{Q}(\mathrm{A}(1))$ - $\mathrm{Q}(\mathrm{A}(2))$, es inferior a 0,333 , e igualmente $\mathrm{Q}(\mathrm{A}(1))$ $\mathrm{Q}(\mathrm{A}(3))$, por lo cual no se cumple la condición 1 del método VIKOR, de ventaja aceptable, por lo cual, como solución al problema de decisión se propone un conjunto de soluciones de compromiso formado por las tres primeras alternativas clasificadas según Q. Pudiendo tomarse como solución la alternativa de muro de escollera que es la mejor clasificada a su vez según el ranking de $\mathrm{S}$.

\section{ANÁLISIS DE SENSIBILIDAD DE LA SOLUCIÓN ÓPTIMA.}


Para determinar la estabilidad de la solución es necesario realizar el análisis de sensibilidad de la metodología cuando se producen variaciones en las condiciones de partida. En este caso vamos a suponer que se producen modificaciones en la poderación de los criterios porque el decisor decide priorizar el precio y el plazo, y dar mayor importancia a la integración paisajística, frente a las necesidades de conservación futuras respecto a la solución óptima obtenida en el apartado anterior.

Repitiendo todo el proceso, se obtiene el vector de pesos $\mathrm{WC}=(0,32 ; 0,31 ; 0,24 ; 0,03 ; 0,09)$, por aplicación de AHP y matriz de comparaciones pareadas,

De esta forma, como resultado del método de decisión de VIKOR, se obtienen los siguientes valores y ranking.

\begin{tabular}{|c|c|c|c|c|}
\hline & $\begin{array}{l}\text { Muro Hormigón } \\
\text { Armado }\end{array}$ & Muro Jardinera & $\begin{array}{l}\text { Muro } \\
\text { Vegetal }\end{array}$ & $\begin{array}{l}\text { Muro de } \\
\text { Escollera }\end{array}$ \\
\hline$S_{\mathbf{j}}$ & 0,8659 & 0,5062 & 0,1319 & 0,3060 \\
\hline $\mathbf{R}_{\mathbf{j}}$ & 0,3155 & 0,2154 & 0,0900 & 0,1511 \\
\hline$\overline{Q_{j}}$ & 1,0000 & 0,5331 & 0 & 0,2541 \\
\hline
\end{tabular}

Tabla 4: Valores de Sj, Rj y Qj, resultado método VIKOR, análisis de sensibilidad

\begin{tabular}{l|c|c|c|}
\multicolumn{1}{c|}{} & $\mathbf{Q}$ & $\mathbf{S}$ & $\mathbf{R}$ \\
\hline Muro Vegetal & 0,0000 & 0,1319 & 0,0900 \\
\hline Muro Escollera & 0,2541 & 0,3060 & 0,1511 \\
\hline Muro Jardinera & 0,5331 & 0,5062 & 0,2154 \\
\hline Muro Hormigón Armado & 1,0000 & 0,8659 & 0,3155 \\
\hline
\end{tabular}

Tabla 5: Ranking de alternativas según método VIKOR, análisis de sensibilidad

Hay que hacer notar que $\mathrm{Q}(\mathrm{A}(1))-\mathrm{Q}(\mathrm{A}(2))$, es inferior a 0,333, como ocurría en el caso anterior, por lo cual no se cumple la condición 1 del método VIKOR, de ventaja aceptable, por ello, como solución al problema de decisión en este caso el conjunto de soluciones de compromiso estaría formado por dos alternativas que son muro vegetal y muro de escollera, soluciones de compromiso que también se obtuvieron en el primer caso, y además son las mejores clasificadas según el ranking de $\mathrm{S}$, en ambos supuestos. Ante este hecho, podemos concluir que el conjunto de soluciones de compromiso obtenido es estable ante pequeños cambios en la ponderación de los criterios y la solución óptima fluctúa poco ante las preferencias del decisor

\section{CONCLUSIONES}


- Disponer para la toma de decisiones de una metodología reduce la subjetividad en el proceso de decisión, al mismo tiempo que constituye una herramienta para sistematizar todo el proceso tanto el análisis de alternativas viables como los criterios que son determinantes.

- El análisis de sensibilidad permite constatar la estabilidad de la solución óptima, y permite validar el método de decisión.

- De igual forma el análisis de sensibilidad permite descartar las soluciones que presentan un comportamiento inestable ante pequeñas modificaciones en las condiciones de partida.

\section{REFERENCIAS}

CORREIA BAPTISTA, J.C., GONÇALVES, E., ANGUlO, L., PEREIRA, M., (2004). Selección de Variables para el Incremento del Poder de Discriminación de los Modelos DEA. Revista de la Escuela en Perfeccionamiento en Investigación Operativa, año XII n ${ }^{\circ}$ 24.

FERNÁNDEZ, G.M., ESCRIBANO, M.C., El Análisis de la Robustez y la Ayuda a la Decisión Multicriterio Discreta, Universidad San Pablo CEU.

MORENO-JIMÉNEZ, J.M., AGUARÓN, J., CANO, F., ESCOBAR, M.T., (1998). Validez, Robustez y Estabilidad en Decisión Multicriterio, Análisis de Sensibilidad en el Proceso Analítico Jerárquico. Rev.R.Acad. Cien.Exact.Fis.Nat. (Esp), Vol. 92, nº4, pp. 387-397.

MUÑOZ, B., ROMANA, M., \& ORDÓÑEZ, J. (2014). Elección de Tipo de Muro en una Autopista Urbana en Servicio por Métodos de Decisión Multicriterio Discretos. Aplicación a la M-40 en Madrid. Obras Urbanas, 34-44, pp. 34-44.

SAATY, T. (1994). How to make a decision: The Analytic Hierarchy Process. Interfaces 24, pp. 19-43.

OPRICOVIC, S., TZENG, G.-H., (2007). Extended VIKOR method in comparison with outranking methods. European Journal of Operational Research, 178, pp. 514 - 529. 